\title{
LA VIOLENCIA ANTES DE LA VIOLENCIA: EL PROCESO DE CONSTRUCCIÓN DE LOS HECHOS VIOLENTOS EN EL FÚTBOL COMO PROBLEMA PÚBLICO EN ARGENTINA
}

\author{
VIOLENCE BEFORE VIOLENCE: THE PROCESS OF CONSTRUCTION \\ OF THE VIOLENT EPISODES IN FOOTBALL AS A PUBLIC PROBLEM IN \\ ARGENTINA
}

\author{
Diego Murzi
}

\begin{abstract}
RESUMEN
Este artículo se propone recorrer el proceso mediante el cual la violencia en el fútbol se convirtió en un problema público en Argentina. Realizando un recorrido por los hechos trágicos que jalonaron al fútbol argentino desde su profesionalización en 1931 hasta el retorno de la democracia en 1983, se analizarán los sentidos que desde la prensa y el Estado le fueron siendo asignados a la violencia particular que tenía lugar alrededor de este deporte. La pregunta que guía al artículo se interroga por qué, pese a haberse registrado a lo largo de gran parte del siglo XX numerosos crímenes violentos y tragedias alrededor del fútbol, recién en 1985 los incidentes en los estadios son objeto de la primera política pública específica elaborada por parte del Estado argentino.
\end{abstract}

Palabras clave: Violencia; Fútbol; Argentina; Problema público.

\begin{abstract}
This article aims to explore the process by which violence in football became a public problem in Argentina. By pointing out the tragic events ocurred in Argentine football from it professionalization in 1931 until the return of democratic life in 1983, we will analyze the meanings that the press and the State were assigned to the particular violence that took place around this sport. The main question is why, despite having registered to throughout much of the twentieth century several violent crimes and tragedies around football, it was not until 1985 that incidents in the stadiums generates the first specific public policy elaborated by the Argentine State.
\end{abstract}

Keywords: Violence; Soccer; Argentine; Public problem.

"Doctor en Ciencias Sociales por la Universidad de Buenos Aires (Argentina). Investigador posdoctoral de CONICET con sede de trabajo en el Instituto de Altos Estudios Sociales (IDAES) de la Universidad de San Martín (UNSAM). Profesor en Universidad Metropolitana para la Educación y el Trabajo (UMET). 


\section{INTRODUCCIÓN}

Actualmente, en el año 2019, la violencia en el fútbol constituye en Argentina un fenómeno que indudable y crecientemente es objeto de la intervención estatal. Existe un considerable número de leyes, normas y reglamentaciones oficiales para regular la acción de las personas que concurren a los estadios de fútbol. Existen agencias de seguridad deportiva en todas las jurisdicciones dedicadas a controlar y neutralizar la eventual violencia en el fútbol. Existen "expertos" estatales que acumulan saberes sobre cómo gestionar al público del fútbol. Existen programas, campañas y acciones desde los distintos niveles estatales, así como desde la Asociación del Fútbol Argentino y desde la Liga destinadas a prevenir la violencia en las canchas. Existe también una percepción generalizada entre la sociedad de que los incidentes en los estadios constituyen un problema social que debe ser afrontado por el Estado. Estos $\mathrm{y}$ otros muchos motivos nos permiten afirmar que la violencia en el fútbol constituye en Argentina un problema público (MURZI, 2019).

Sin embargo, hasta la década de 1980, la violencia en el fútbol no constituyó en Argentina un problema que ameritara un tratamiento específico por parte del Estado. La única dimensión del espectáculo futbolístico que estaba medianamente reglamentada era la que se refería a la infraestructura de los estadios, y esto sólo en determinadas provincias y jurisdicciones. Ni la gestión policial de los espectadores ni las transgresiones que ocurrían en el espacio del estadio eran todavía objeto de reglamentaciones jurídicas que atendieran al fútbol como un evento de conflictividades particulares.

Si bien en la lista negra del fútbol argentino se contaban hacia los años 1980 más de cien muertes (ROMERO, 1985), centenares de heridos, innumerables incidentes y hasta un par de tragedias, que daban cuenta de un tipo de violencia particular que se producía alrededor del fútbol, la lectura estatal del problema insistió durante gran parte del siglo XX en considerar al fenómeno deportivo -del cual el fútbol constituía la punta de lanza- como divorciado del resto de los campos sociales. Esta lectura constituyó un obstáculo para el tratamiento del problema, en tanto daba por presupuesto que el campo deportivo debía autorregularse con sus propias normas y reglamentos, incluyendo en ello lo que se refería a la violencia de sus protagonistas y del público asistente.

A partir de ese escenario, las preguntas que guían este artículo son las siguientes: ¿qué ocurrió para que esa perspectiva cambie y la violencia en el fútbol comience a ser percibida como un problema público que requiere la atención de los actores gubernamentales, como sucede hoy en día (MURZI, 2019)? ¿Cuándo se produce ese tránsito en la interpretación del fenómeno por parte del Estado argentino, que pasó a identificar al fútbol como un espacio de conflictividades particulares que deben ser atendidas con reglamentaciones específicas? ¿En qué momento las agendas sociales, las agendas mediáticas y las agendas políticas confluyeron en la designación de los hechos de violencia alrededor del fútbol como constitutivos de un tema problemático que requería acciones del Estado? ¿Qué sentidos particulares y orientaciones determinadas le impregnaron la prensa y el Estado argentino al fenómeno de la violencia en el fútbol a través de las distintas lecturas que a través del tiempo fueron realizando del peligro y el conflicto asociado a la acción de los simpatizantes que asistían a los estadios?

Con el objetivo de problematizar estos interrogantes, el artículo está planteado siguiendo un orden cronológico en el cual los conectores de los distintos momentos históricos son las muertes y las tragedias ocurridas a lo largo del siglo XX en el marco del fútbol argentino. El punto de llegada es la sanción de la Ley no 23.184 en el año 1985, que constituye la primera legislación específica referida al espectáculo futbolístico en Argentina.

Actualmente existe un consenso teórico acerca de que los problemas públicos no son una derivación natural de condiciones objetivas. Sin embargo, coincidimos con Galar (2017) en la necesidad de contemplar al sustento estructural de los problemas. Para que un problema público se constituya como tal deben manifestarse "experiencias sociales, malestares colectivos o eventos estructurales que soporten una preocupación compartida" (GALAR, 2017): el análisis de dichas condiciones -múltiples, heterogéneas y escurridizas- para el caso de la violencia en el fútbol es lo que reconstruiremos y pondremos de manifiesto en este artículo. 


\section{LA GÉNESIS DE LA VIOLENCIA EN EL FÚTBOL}

Los incidentes, las peleas, los heridos e incluso los muertos son elementos que forman parte del paisaje del fútbol argentino desde los inicios de este deporte en Argentina, y existen registros de prácticas violentas en los partidos incluso antes de la profesionalización del fútbol en el año 1931 (FRYDENBERG, 2010; FABBRI, 2008; PALOMINO Y SCHER, 1988). A diferencia de lo ocurrido en otros países en los cuales el fútbol también adquirió una centralidad cultural, social y política equiparable a la que posee en nuestro país 1 , los casos de violencia en los estadios argentinos se presentaron durante la primera mitad del siglo XX con considerable recurrencia (ROMERO, 1985; ALABARCES, 2015; ARCHETTI, 1992). El asesinato del simpatizante uruguayo Pedro Demby a manos de un hincha argentino en 1924, los crímenes de los hinchas López y Munitoli como consecuencia de la represión policial en el estadio de Lanús en 1939 y la muerte de 9 espectadores en el estadio Monumental de River Plate en la llamada "catástrofe de la Puerta 11" en 1944, son antecedentes que jalonan durante las primeras décadas de 1900 una prematura pero sostenida relación de la violencia con el fútbol vernáculo (ROMERO, 1985).

Entre 1920 y 1950 el fútbol se convierte paulatinamente en uno de los mayores espectáculos de masas en Argentina (FRYDENBERG, 2010; ALABARCES, 2002). En esos años, como sostiene Szlifman (2010) a partir de un análisis de los tres principales periódicos nacionales de la época - Clarín, Crítica y La Nación -, la concepción del fútbol que primaba en la prensa era la del espectáculo como espacio de esparcimiento, de liberación y de disfrute para los crecientes sectores medios y populares. Pero, a su vez, también influía en los discursos periodísticos la idea de la práctica deportiva como vehículo del desarrollo moral individual y colectivo, propia de la Inglaterra de fines del siglo XIX (ELIAS, 1986), a partir de la cual el deporte era considerado como un espacio de reglas, comportamientos y valores que debían ser respetados y promovidos. Desde ese marco interpretativo, tal como lo señalaron Elías y Dunning

\footnotetext{
${ }^{1}$ Pensamos en Inglaterra, en Italia, en Brasil, en Alemania, países a los que Patrick Mignon (1998: 87) identificó junto a la Argentina como "las grandes naciones del fútbol".
}

(1994), la violencia que tenía lugar en el mundo del deporte aparecía como un agente exógeno, inesperado y brutal que venía a perturbar los valores positivos asociados al juego: tolerancia, respeto, solidaridad, cooperación, civilidad. Como sostiene Szlifman, “... en los discursos periodísticos, lo violento aparece como un desplazamiento, considerado así como insólito, exagerado, ajeno y extemporáneo" (2010: 4).

El propio núcleo del fútbol como moderno espectáculo de masas capaz de vehiculizar además los valores probos del deporte es perturbado frente a los hechos de violencia que ocurren en los estadios. Para Szlifman, "la violencia transforma la propia concepción del espectáculo y lo convierte en una cosa distinta a su objeto inicial" (2010: 4), como lo refleja la cobertura periodística de las muertes de Demby en 1924 y de López y Munitoli en 1939: "la violencia transforma el espíritu del fútbol" (La Nación 4/11/1924) y la "fiesta del deporte" se convierte "en un escenario sangriento" (Crítica 16/5/1939). Estas concepciones aparecen tanto en los editoriales de los diarios como en testimonios que se recogen allí desde los lugares de poder, "tanto del poder político como del deportivo" (Szlifman, 2010).

Hasta mediados de los años 1950, entonces, la violencia no era un dato que los discursos mediáticos, políticos ni deportivos situaran como un elemento constitutivo del fútbol argentino. Se trataba más bien de algo inesperado, infrecuente y subrepticio, producto de accidentes, de la mala fortuna o del accionar de algunos "revoltosos" (La Nación 15/5/1939) o "indisciplinados" (La Nación 17/5/1939). Si bien sucedía - como lo testimonian los 15 muertos en los estadios ocurridos entre 1922 y 1955 - , la violencia en el fútbol aún no merecía la atención del gran público ni de los medios de comunicación, y menos aún del Estado a través de sus aparatos legislativos o judiciales.

El escenario -al menos a nivel simbólicocambia a partir de dos hechos luctuosos: la muerte de los hinchas Alberto Linker en 1958 y Héctor Souto en 1967. Con esas muertes se inaugura una de las características que tendrán las respuestas públicas a la violencia en el deporte en Argentina: el hecho de estar fuertemente vinculadas a las muertes que tienen lugar en los estadios de fútbol (SEGURA, MURZI \& NASSAR, 2018). A lo largo de los últimos 50 años se observará una constante reciprocidad entre las muertes 
y las respuestas estatales en forma de política pública, movimiento que David Garland (2006) denomina de "acting out" y Pablo Alabarces (2004) como "respuestas espasmódicas".

Ese carácter reactivo de las formas de control estatales de la violencia en el fútbol es uno de los elementos que será puesto en discusión a lo largo de este artículo. Existe un consenso entre gran parte de los autores argentinos que han abordado el problema de la violencia en el fútbol (ALABARCES, 2004; GARRIGA, 2013; SUSTAS; 2013; SEGURA \& MURZI, 2015; CABRERA, 2016) en señalar el carácter de "acting out" de las políticas públicas de control de la violencia en el fútbol a partir del retorno de la democracia en 1983. Nosotros, en nuestro propio trabajo de tesis de doctorado (MURZI, 2019) reafirmamos esa hipótesis para buena parte de dicho período. Pero, al mismo tiempo, nos planteamos a su vez una pregunta complementaria: ¿por qué en el período histórico previo al retorno de la democracia en 1983 no existían respuestas estatales en forma de política pública frente a los hechos de violencia en los estadios, cuando éstos ya se venían produciendo desde décadas atrás de manera recurrente? Dicho de otro modo: la violencia en el fútbol no es un producto de los años 1980 ni del retorno de la democracia en Argentina; sin embargo, el abordaje legislativo por parte del Estado argentino del fenómeno de la violencia en los estadios sí lo es.

\section{Dos muertes centrales y la emergencia de las "barras"}

Con los asesinatos de Linker y Souto, dos adolescentes que aún no habían cumplido los 20 años, se inicia el derrotero en el cual las muertes en los estadios son disparadores de cambios en la concepción, interpretación o gestión del fenómeno de la violencia en el fútbol. En estos dos casos en particular, sin embargo, las transformaciones no ocurrieron a nivel de la acción estatal sino predominantemente en relación a la lectura que tanto los medios de comunicación como el propio Estado hicieron de los hechos.

El caso de Linker, asesinado en el estadio de Vélez debido a una granada de gas policial supuestamente arrojada con el objetivo de dispersar incidentes ocasionados por los hinchas locales que estaban ocurriendo en la tribuna, va a dar cuenta de la emergencia de los hinchas organizados como sujeto. Esa emergencia es designada por la prensa con el mote de "barras fuertes". Como señala Amílcar Romero (2009),

Tres o cuatro días después del hecho, en el diario La Razón aparece el concepto de 'barras fuertes' como algo ya conocido en el fútbol, que todos los dirigentes sabían que existían y dónde paraban, y que son el paso previo a la aparición de las barras bravas (p. 2).

La caracterización es trascendente en tanto se designa por primera vez a los hinchas como un sujeto colectivo y medianamente organizado. La hipótesis que explicaba los hechos violentos únicamente a partir de la acción aislada de un simpatizante exaltado, va a comenzar a dar lugar a una nueva interpretación, ligada a los hinchas como grupo organizado. De todas formas, aún prevalecen - y esta narrativa será una constante en la prensa deportiva argentina incluso hasta nuestros días - las explicaciones asociadas a la acción individual y a lo salvaje, no-evolucionado - individuos con "sentimientos primitivos", como califica el diario La Nación del 25/10/1958.

El caso Linker no crea un nuevo sujeto, pero le da entidad a partir de ser nombrado por la prensa: los grupos de hinchas medianamente organizados existían alrededor de los equipos de fútbol desde décadas atrás (FRYDENBERG, 2010; PALOMINO \& SCHER, 1988), pero es su designación bajo el mote de "barras fuertes" que los empieza a configurar como un nuevo sujeto. En pocos años, el nombre de "barras fuertes" irá mutando hacia el de "barras bravas" que se utiliza actualmente.

Esta caracterización es trascendente si consideramos con Sodo (2010) que "no es posible leer la historia del fútbol argentino, y menos todavía en clave violencia, sin tener en cuenta el papel decisivo que en ella han tenido los medios de comunicación" (p. 66). Uno de nuestros argumentos es que los ciclos de políticas públicas de gestión de la violencia en el fútbol en Argentina se encadenan de forma estrecha con los humores sociales que existen en torno a la cuestión. Y entendemos, siguiendo a Garland (2006), Kessler (2008) y Sozzo (2009), que esos humores sociales están fuertemente vinculados con los sentidos que los medios de comunicación vehiculizan respecto a los problemas sociales. Observar las interpretaciones 
de los medios de comunicación es entonces necesario para comprender el desarrollo del tratamiento estatal del problema de la violencia en el fútbol a lo largo del tiempo (ALABARCES, 2015).

Si la muerte de Linker fue relevante porque generó un cambio en la mirada de la prensa sobre los hinchas, el asesinato del joven Héctor Souto en 1967 lo fue aún más para nuestro argumento, en tanto propició la primera toma de posición estatal respecto al tema. Numerosos trabajos socio-antropológicos coinciden en considerar la muerte de Souto como una bisagra que marca un antes y un después en torno a la historia de la violencia en el fútbol argentino (ALABARCES, 2004; ROMERO \& ARCHETTI, 1994; GARRIGA, 2013; MURZI, ULIANA \& SUSTAS., 2011, SEGURA, MURZI \& NASSAR, 2018). Los motivos para establecer esta división tienen que ver con las condiciones en que se produjo esa muerte, asociada por primera vez a un tipo de violencia cuyas causas responden puramente al enfrentamiento entre hinchas.

Souto es asesinado a golpes en la tribuna de Huracán durante un partido que el club local juega ante Racing Club, equipo del cual la víctima era simpatizante. El "error" de Souto consistió en ingresar de forma equivocada a la tribuna contraria, y tras una pelea donde intentó defender a un amigo que estaba siendo atacado, fue masacrado por el núcleo duro de la hinchada de Huracán. Según Romero y Archetti (1994), la muerte de Souto es la primera que puede asociarse al fenómeno de las "barras bravas" "modernas", con algunas de las características que éstas poseen en la actualidad. Pero, además, esta muerte marca el paso de un tipo de violencia simbólica a un tipo de violencia real entre los hinchas argentinos (ROMERO \& ARCHETTI, 1994; ALABARCES, 2004).

La muerte de Souto es de gran importancia porque habilita por primera vez una interpretación estatal del fenómeno de la violencia en el fútbol. Esa lectura proviene del poder judicial, a través del juez Moras Mom quien incluyó el término "barra brava" al momento de elaborar la sentencia para los acusados del crimen:

Que el diciente quiere dejar aclarado lo que quiere decir por barra brava: un grupo perfectamente determinado que concurre todos los domingos no a presenciar el partido y sí, en cambio, a provocar todo tipo de depredaciones y agresiones físicas a los demás concurrentes al espectáculo; que dicha barra brava, cuando baja de la tribuna, lo hace con el propósito de dar leña a cualquier persona, llegando incluso, como en este caso, a cometer un homicidio; que dichas barras bravas portan banderas, bombos, estandartes y otros elementos de otros clubes, los que consiguen en luchas con barras similares y que guardan como trofeos, que siempre son comandados por sujetos de gran predicamento y amparados por los clubes que son los que costean los viajes y les guardan en las sedes sociales banderas, bombos, sombrillas y demás trofeos; que siempre concurren al mismo lugar y se encuentran perfectamente organizadas para sus quehaceres de provocación y agresión" (ROMERO, 2009: 4).

De esta manera, el término "barras bravas" es empleado en un texto estatal para ilustrar a un sujeto nuevo: se trata de grupos de hinchas organizados, que incluyen entre su repertorio de acción a las prácticas violentas, y que tienen vínculos con los dirigentes de los clubes. Sin embargo, el término no se expandiría de la forma en que lo conocemos ahora sino hasta más de 15 años después. En los discursos de la prensa va a seguir predominando la idea del desvío moral, de la "incultura" y de las acciones irracionales aisladas de un puñado de personas, presentadas como ajenas y extemporáneas al gran colectivo del resto de los simpatizantes, caracterizados como "espectadores pasivos" (La Nación 11/4/1967), o "gente decente" (Clarín 11/4/1967). La Nación (14/4/1967) hablará de "graves episodios de incultura", "desbordes de pasión" y "revanchismo de la incivilización" (SZLIFMAN, 2010).

Las muertes de Linker y Souto reflejan la irrupción de un fenómeno social que, si bien se hallaba presente desde los inicios del fútbol en nuestro país, se revela repentinamente capaz de cobrarse víctimas fatales y de funcionar como disruptor de la fiesta popular que encarnaba el fútbol. La prensa y el poder judicial traducen ese fenómeno en conceptos, delineando con sus respectivos tratamientos la génesis de un sujeto social nuevo y peligroso, encarnado en los hinchas de fútbol organizados. Sin embargo, esos sucesos no alcanzan para que el Estado preste atención al problema de forma específica. La violencia en el fútbol es percibida estrictamente como un problema de orden público equivalente al de otras manifestaciones masivas. 


\section{EN EUROPA, UN PROBLEMA DE “ORDEN PÚBLICO” ORDINARIO}

A fines de la década de 1960, los incidentes entre hinchas de fútbol también irrumpen como un fenómeno novedoso en Inglaterra, país central en el mapa futbolístico del siglo XX y espejo para el mundo del fútbol global. Allí, los cambios en los comportamientos de los hinchas llaman tempranamente la atención de la comunidad científica - aún antes que la del Estado - y se producen los primeros trabajos sobre el fenómeno del hooliganismo (TAYLOR, 1969, 1971; CLARKE, 1976; MARSH, 1975, INGHAM, 1978), nombre que adquiere en Inglaterra el problema de la violencia en el fútbol.

En el plano del control social, a pesar de la expansión rápida del hooliganismo y de la creciente gravedad de los incidentes, el fenómeno es considerado por las autoridades como un problema de orden público ordinario. Su control se apoya sobre marcos normativos generales y estrategias de acción policial ordinarias, y su represión se realiza en virtud de disposiciones del Código Penal y a partir de leyes y estatutos de "ofensas" previstas por la ley de orden público en general (TSOUKALA, 2009).

Esto no significó que el temprano hooliganismo fuese tomado de manera laxa en Inglaterra. Por el contrario, fue objeto de una represión cada vez más firme por parte de la policía y de la Justicia, sólo que desprovisto de un cuadro normativo específico. En los años posteriores, el fenómeno se agrava y se expande por toda Europa, sumando a su aspecto emocional y espontáneo, una planificación y organización de parte de los hinchas cada vez más perfeccionada.

Por supuesto que en cada país el fenómeno del hooliganismo fue percibido y tratado de diferente manera, dependiendo de las particularidades locales. En ese sentido, Tsoukala (2009) sostiene que en aquellos países que vivieron largos conflictos políticos bajo un clima de agitación sociopolítica intensa en los años 1960, como Italia o Grecia, se produjo una banalización de la violencia en el fútbol por parte de la prensa y las autoridades estatales, generando que el hooliganismo no fuese percibido de forma tan grave como en los países con climas sociales más estables, como Inglaterra.

Esta hipótesis podría ser interesante para pensarla en función del caso argentino, en la medida que entre la muerte de Souto en 1967 y el retorno del proceso democrático en 1983 el fenómeno de la violencia en el fútbol pareció entrar en un impasse, al menos desde su representación oficial como problema. Si en Italia, por ejemplo, las autoridades fueron en los años 1970 más moderadas, incluso indulgentes frente al hooliganismo por considerarla un tipo menor de violencia juvenil, no es difícil imaginar un paralelo con lo que ocurría en nuestro país entre fines de los años 1960 y la primera mitad de los años 1970, cuando la violencia política se vuelve moneda corriente y sus principales intérpretes son los jóvenes urbanos. También es posible que la violencia en el fútbol quedase subsumida en otros "blancos" elegidos por el sistema de control oficial; allí, el ministro José López Rega promulga dos leyes casi en simultáneo, la famosa ley antisubversiva $\left(\mathrm{N}^{\circ}\right.$ 20.840) y la penalización por tenencia de drogas $\left(\mathrm{N}^{\circ}\right.$ 20.771), unificando así la juventud, el consumo de drogas y la subversión en un mismo locus de sentido (CAMAROTTI,2017).

Aclaremos que esta hipótesis la estamos pensando relacionada a la circulación de discursos oficiales sobre la violencia en el fútbol, particularmente los de la prensa y los del Estado. En la realidad de los estadios, en las canchas de cada fin de semana, tanto las prácticas violentas como la organización de los grupos de hinchas más radicales siguieron aumentando a ritmo leve pero sostenido durante la década de 1960 y 1970 (ALABARCES, 2004; ROMERO, 1985).

\section{La “Tragedia de la Puerta 12" y el primer antecedente normativo}

La mayor catástrofe de la historia del fútbol argentino ocurrió en 1968, meses después de la muerte de Héctor Souto, cuando 71 personas perdieron la vida aplastadas al intentar salir por un ingreso no habilitado del estadio de River Plate, en la llamada "Tragedia de la Puerta 12". Ese día, en un partido entre Boca y River en el Estadio "Monumental", al finalizar el encuentro los hinchas de Boca que descendían en masa por las escaleras del estadio se toparon con que la puerta número 12 estaba cerrada. Las responsabilidades del "olvido" nunca quedaron determinadas, pero sus efectos fueron devastadores: la marea humana que bajaba por las escaleras se amontonó frente a las rejas y el propio peso de la gente que seguía bajando sin advertir el problema generó que perdieran la vida 
aplastadas 71 personas y que 113 resultaran heridas. El promedio de edad de los muertos fue de 19 años.

Este hecho marcó profundamente la memoria colectiva futbolera nacional, y fue objeto de varias investigaciones 2 y de otras tantas especulaciones. Sin embargo, pese a la magnitud de los acontecimientos y a lo dramático de su desenlace, esta tragedia no implicó una transformación sustancial en el modo de habitar los estadios por parte de los hinchas en Argentina, ni tampoco motivó grandes cambios a nivel de las formas de control social alrededor del fútbol.

La principal consecuencia que dejó la Tragedia de la Puerta 12 fue la sanción de la primera normativa de la Ciudad de Buenos Aires sobre seguridad en estadios de fútbol: la Ordenanza Municipal n ${ }^{\mathrm{a}}$ 24.225, del año 1969. Se trata del primer texto legislativo exclusivamente referido al fútbol producido en dicha jurisdicción. Esta norma, que años más tarde fue derogada, determinaba las condiciones edilicias y de infraestructura de los estadios, regulaba el ingreso de objetos y personas, estacionamiento, capacidad de ocupación y servicios de seguridad con los que debía contar cada estadio ubicado dentro de la Capital Federal.

La mayor parte de los artículos de la Ordenanza $\mathrm{n}^{\mathrm{a}} 24.225$ referían a las condiciones edilicias: establecía la capacidad de las tribunas, regulaba los ingresos y egresos, el trazado de pasillos y escaleras y los tipos de molinetes y bretes que debían utilizarse. La necesidad legislativa de ordenar el espacio del estadio a través de medidas de ese tipo puede leerse como una consecuencia directa de la Tragedia de la Puerta 12, en donde las fallas de organización e infraestructura fueron señaladas como los causales del hecho.

Pero también se incluyen en la normativa situaciones que no sólo involucran a las estructuras materiales, sino también a las personas que concurren a los partidos. En el artículo $5^{\circ}$, donde el Estado municipal se atribuye la potestad de suspender el partido de fútbol en caso de considerarlo necesario, se hace referencia al desalojo de personas que "alterasen el orden" o "perturbaran el normal desarrollo del espectáculo". Mientras que en el artículo 9, rotulado como "Orden y Comodidad" se determina que "No será permitido el acceso o permanencia de personas en

${ }^{2} \mathrm{Al}$ respecto se recomienda el documental "Puerta 12" de 2008 dirigido por Pablo Tesoriere. estado de ebriedad ni las que lleven bultos u objetos que puedan ser arrojados". En estas menciones se refleja la misma cosmovisión que, como vimos, la prensa movilizaba sobre la violencia alrededor del fútbol: lo que prima es la idea de un orden colectivo extendido y "natural", frente al cual los incidentes son percibidos como raros, infrecuentes o producto de sujetos con conciencias alteradas (alcoholizados).

El peligro para los espectadores que asisten a un partido de fútbol en la Ciudad de Buenos Aires en 1969, nos dice esta ordenanza, proviene fundamentalmente de las malas condiciones infraestructurales de los estadios. Esto queda reforzado al analizar el apartado de la norma titulado "Servicio de Seguridad". En el mismo se hace referencia a la obligatoriedad de que el estadio cuente con una sala de primeros auxilios médicos, con un servicio contra incendio y con señalización exhaustiva de todos los ingresos y egresos. La seguridad aquí es pensada únicamente respecto a los peligros del escenario que alberga a las personas y no a la acción de estas dentro de ese escenario.

Lejos estamos en 1969 de encontrar una conceptualización del término "seguridad" similar a la que se fue adoptando en las décadas posteriores. Esta "seguridad" se encuentra mucho más cerca del término anglosajón "safety" -que hace referencia a la seguridad frente a riesgos de origen técnico, laboral o natural, y se traduce al español como "protección" en el uso extendido, o "bienestar" en la literatura especializada sobre organización de eventos masivos -, que a "security", término que refiere más a la seguridad física frente a daños ocasionados por otras personas (GIULIANOTTI \& KLAUSER, 2010).

Sin embargo, lo que resalta en esta reglamentación es sobre todo lo que esquiva: la responsabilidad de los organizadores del espectáculo en términos penales. Si bien fue reconocido en el proceso por la tragedia de la Puerta 12 que hubo negligencia del club River Plate, el output de política pública que sucedió como respuesta al hecho no se trató de una ley penal que castigase a los organizadores de espectáculos futbolísticos, sino sólo una normativa municipal sobre condiciones edilicias. Para observar el contraste basta recordar las consecuencias de la tragedia de la discoteca Cromañón en 2004 - la mayor tragedia en términos de víctimas de la historia de los espectáculos masivos de la Ciudad de Buenos Aires 
-, donde los organizadores fueron condenados como responsables primeros de los hechos. En la Puerta 12, más allá de tratarse de una época diferente a la de Cromañón, aparece algo que va a perdurar en el tiempo como especificidad del fútbol en Argentina: la responsabilidad de los organizadores de los partidos -léase la Asociación del Fútbol Argentino y los clubessiempre estará matizada y escamoteada.

La importancia de la Ordenanza $n^{\mathrm{a}} 24.225$ reside en varios puntos. En primer término, se trata de la primera normativa de la Ciudad de Buenos Aires que atiende al fútbol como un caso especial, distinto al de otros eventos masivos. En segundo lugar, porque da cuenta del paradigma vigente de la época previa a los años 1980 en cuanto a la concepción de la peligrosidad en el fútbol: se trata más de una cuestión infraestructural que social o colectiva, y por ende a los hinchas hay que protegerlos y no "defenderlos". Y en tercer orden, porque inaugura el ciclo de políticas públicas que son sancionadas como respuesta inmediata a un hecho de violencia, mecanismo que, como señalamos, será constante y habitual en la historia de las acciones estatales de prevención y control de los incidentes en el fútbol.

En términos de control social, la tragedia fue absorbida conceptualmente por el limbo de la "fatalidad", más vinculada al orden de lo accidental que a las lógicas de funcionamiento de los estadios argentinos, en donde crecientemente ya se reproducía aquello que Juan Sodo (2013) caracterizó como "ambientes de violencia". El rol de los hinchas no fue puesto en cuestión tras la tragedia. Algunas investigaciones (ALABARCES, 2004) señalan la responsabilidad de la policía en el hecho, pero lo cierto es que, a diferencia de lo que ocurriría en los años 1980 en Europa tras la tragedia de Heysel y en el Reino Unido tras el desastre de Hillsborough, la tragedia de la "Puerta 12" no funcionó como disparador de grandes transformaciones organizacionales, políticas, económicas ni del orden de la seguridad en el futbol argentino.

De hecho, al igual que lo que sucedía en el Reino Unido en los años 1960 y 1970 (ARMSTRONSG \& HARRY, 1991; BRAUN \& VLIEGENTHART, 2008), los hechos de violencia ligados al futbol en nuestro país fueron tratados en esos mismos años a través del Código Penal. Esto se cristaliza con la sanción de la Ley del Deporte ( $\left.\mathrm{N}^{\circ} 20.655\right)$ de 1974, donde el
Estado asume la obligación de: "velar por la seguridad y corrección de los espectáculos deportivos", pero sin especificar la manera en que cumpliría esa obligación.

En dicha Ley, dentro del apartado de delitos vinculados al deporte aparecen únicamente el soborno o arreglo de partidos y el dóping, es decir, transgresiones del principio de igualdad que son la base del deporte moderno (ELÍAS, 1986). Se trata de delitos ligados a la competencia deportiva y no a la organización general del deporte o a los espectadores.

La separación entre los delitos deportivos y el resto de los delitos en las atribuciones de la Ley del Deporte refuerza la concepción de la violencia en el fútbol que venimos señalando como hegemónica para los años previos a 1983. Esta se basa en una idea que consiste en separar al mundo del deporte de otros mundos sociales, como si el fenómeno deportivo fuese ajeno a las demás manifestaciones sociales, políticas, económicas y culturales (BOURDIEU,1988; BROHM, 1982). La Ley del Deporte asume solamente la represión de los delitos deportivos, y deja fuera del ámbito de criminalización a los espectadores y organizadores bajo la idea de que los delitos y transgresiones cometidos por esos actores se pueden tratar con las figuras que incluye el Código Penal: lesiones, homicidios, asociación ilícita, etc.

\section{LOS AÑOS 1980, EL PUNTO DE LLEGADA}

Como vemos, hasta la década de 1980 la violencia en el fútbol no constituyó en Argentina una cuestión que ameritara un tratamiento específico por parte del Estado, ni desde el punto de vista jurídico ni tampoco desde la generación de política públicas. La única dimensión del espectáculo futbolístico que estaba medianamente reglamentada era la que se refería a la infraestructura de los estadios, y esto sólo en determinadas provincias y municipios. Ni la gestión policial de los espectadores ni las transgresiones de simpatizantes que ocurrían en el espacio del estadio eran todavía objeto de reglamentaciones que atendieran al fútbol como un evento de conflictividades particulares.

Retomando la pregunta central de este artículo nos interrogamos: ¿qué motivó el cambio perspectiva para que la violencia en el fútbol comience a ser percibida como un problema público? ¿Por qué las 
agendas sociales, las agendas mediáticas y las agendas políticas confluyen en la designación de los hechos de violencia alrededor del fútbol como constitutivos de un tema problemático que requiere acciones del Estado?

Nuestro argumento sostiene que, a diferencia de lo ocurrido en Europa en general y en el Reino Unido en particular a partir de la "tragedia de Heysel" en 1985, el motor del cambio de perspectiva que se operó en las lecturas estatales del fenómeno no ocurrió en Argentina a partir únicamente de un hecho puntual o un gran acontecimiento trágico. En Argentina, el cambio de perspectiva se asemeja más al punto de llegada de un proceso de varios años de duración, claramente jalonado por sucesos trágicos en las canchas, pero también, y por sobre todo, fuertemente apuntalado por factores de otra índole.

Entre esos factores hay tres que identificamos como los más determinantes. El primero es el cambio operado en los grupos de hinchas organizados, cuyo perfil evolucionó, entre aquellas "barras fuertes" del asesinato de Linker en 1958 y las "barras bravas" de los años 1980, hacia formas donde la violencia fue adquiriendo cada vez más preponderancia. En esa mutación del perfil de los grupos de hinchas, un nuevo elemento fue ganando espacio en la caracterización de éstos por parte del discurso mediático: la vinculación de las "barras bravas" con el mundo del delito. Como señala Sustas (2013), hasta principios de los años 1980, en los medios de comunicación se reproduce un discurso que liga a las prácticas violentas con lo patológico e irracional. Pero a partir de allí también se sumará el elemento delictual, haciendo que lo patológico y lo criminal convivan en la caracterización de las cada vez más extendidas "barras bravas".

El segundo factor es la emergencia embrionaria de lo que Stanley Cohen (1972) denominó “pánicos morales", asociados a la caracterización que venían haciendo los medios de comunicación de los hinchas de fútbol. De la mano de la introducción de elementos vinculados al crimen, a la desviación y a la violencia, tanto el espectáculo futbolístico como los grupos de hinchas organizados comienzan a ser leídos por los medios de comunicación como focos de amenaza y peligro. Esa lectura tendrá gran pregnancia en los imaginarios de los actores gubernamentales, como lo reflejan los debates parlamentarios alrededor de la sanción de la Ley 23.184 "Régimen Penal y
Contravencional para la Violencia en Espectáculos Deportivos" en 1985.

El tercer factor que incidió en el cambio de la concepción estatal respecto del fenómeno de la violencia en el fútbol es, a nuestro entender, el más decisivo pero el menos problematizado hasta el momento por la literatura especializada. Tiene que ver con el cambio social y político operado en Argentina durante la última parte de la década de 1970 y la primera parte de la de 1980. Con el progresivo declive de la violencia política que caracterizó a la década de 1970, la restitución del proceso democrático vio emerger nuevas violencias y conflictividades sociales que rápidamente se incorporaron a la agenda política como nuevos fenómenos a los que el Estado debió dar respuesta (KESSLER, 2009). La seguridad se convertiría paulatinamente en un objeto de gobierno, y a la noción de "orden público" -asociada a las de "seguridad interior" y "seguridad nacional" tan repetidas en los años del terrorismo de Estado-, se le opone la de "seguridad ciudadana", vinculada a las formas de gobierno de la seguridad propias de un moderno Estado de Derecho (GALVANI, 2010).

Estos tres factores que mencionamos deben pensarse encadenados e interdependientes entre sí. La incorporación de la dimensión criminal en los grupos organizados de hinchas moldea un nuevo "sujeto peligroso", que es recuperado y procesado por los discursos simplificadores y estigmatizantes de la prensa, dando lugar a la generación de incipientes "pánicos morales" en torno a los hinchas de fútbol. Esto, sumado a un contexto de reacomodamiento de las fuerzas sociales y políticas al calor de la nueva democracia, y a una redefinición de los problemas públicos en general y de las violencias en particular, dio lugar a un cambio en la perspectiva estatal respecto al problema de la violencia en el fútbol.

Por todo lo señalado, para nuestro argumento vamos a considerar a la vuelta de la democracia en 1983 como la antesala del inicio de las políticas públicas de gestión de la violencia en el futbol en Argentina. El verdadero mojón que marca el inicio de esas políticas públicas activas y propositivas es la sanción de la Ley $N^{\circ} 23.184$ el día 25 de junio de 1985, que constituye el primer antecedente legislativo referido a la gestión de la violencia en el fútbol. 


\section{LOS NUEVOS PROBLEMAS DE LA DEMOCRACIA. LEY N.o 23.184}

Difícilmente se pueda comprender el derrotero del campo jurídico, del campo penal y del campo de la seguridad en las décadas de 1980 y 1990 en Argentina al margen de los efectos que el terrorismo de Estado producto del último gobierno militar generó en las instituciones y en el conjunto social. El proceso de democratización implicó una revalorización del acceso a la justicia, de la capacidad de los tribunales para garantizar derechos y de las herramientas legislativas, en tanto indicadores relevantes de la calidad democrática (JELIN,1995).

En este sentido, uno de los rasgos del proceso de construcción de "nueva" ciudadanía (DE SOUSA SANTOS, 2010; TOURAINE, 1995) bajo el marco del Estado de Derecho fue la creciente regulación de las prácticas y conductas de los ciudadanos a través de los aparatos legislativos y judiciales (JELIN,1995; ZIMMERMAN,1999). Frente al fantasma reciente de la violencia institucional, las prácticas represivas y el autoritarismo, las "nuevas" violencias civiles que emergieron a la salida de la dictadura -con el delito a la cabeza- fueron rápidamente objeto de gran atención por parte del nuevo aparato estatal democrático y de los medios de comunicación. La violencia que ocurría alrededor del fútbol fue una de ellas.

Según la ONG "Salvemos al Fútbol”, en los años 1983 y 1984 se registraron 11 muertes relacionadas al fútbol en Argentina. La misma cantidad de fallecidos que existió entre la Tragedia de la Puerta 12 en 1968 y el año 1982. Es decir que, en los dos primeros años del regreso de la democracia, murieron la misma cantidad de personas en el fútbol que en los 15 años previos.

Ese salto cuantitativo resulta muy llamativo, y son varias las hipótesis a las que podemos echar mano para explicarlo. La menos creíble tiene que ver con una supuesta efectividad en el control del fenómeno por parte de los gobiernos entre 1968 y 1983, en su mayoría militares, que habría explotado tras el "aflojamiento" de los controles luego de la retirada del último gobierno de facto. Una hipótesis más interesante es la de Alabarces (2004), quien postula que las "barras bravas" heredan prácticas y formas de los grupos clandestinos de tareas de la última dictadura, cuyo despliegue acrecienta el nivel de violencia en los hechos que las involucran.
También es sugerente la lectura de Sustas (2011), quien a partir de identificar que el 30\% de las muertes de la década de 1980 se enmarcan en enfrentamientos de hinchas con la policía, considera a la represión policial en el fútbol como una faceta en que toma forma la violencia institucional en esa década, y como evidencia de la supervivencia de las prácticas represivas del gobierno militar.

Pero la explicación más certera, creemos, tiene que ver con la cuestión de la visibilidad. Visibilidad de las cifras oficiales en primer término: si la desaparición de personas y el ocultamiento de cuerpos constituyó una práctica estatal extendida durante el último gobierno militar, no es llamativo que los muertos del fútbol también hayan sido ocultados, negados o escamoteados. El altísimo desfasaje entre las cifras "oficiales" y las cifras "reales" de los muertos en general nos hacen suponer con cierto grado de certeza que, durante esos años, los muertos del fútbol quedaron en la cuenta de la "cifra oscura" en términos criminológicos.

$\mathrm{Y}$, en segundo término, visibilidad también de las prácticas y los nuevos sujetos: los grupos de hinchas organizados se consolidan definitivamente como un actor central del fútbol, en particular las "barras bravas". Esto quedó en evidencia con la muerte de Aníbal "Matutito" Taranto, integrante de alta jerarquía de la "barra brava" de River, asesinado en un tiroteo con la "barra" de Boca en octubre de 1983. En su análisis de la cobertura que los medios de comunicación hicieron de los principales casos de violencia en el fútbol a lo largo del siglo pasado, Szlifman postula que, "mientras en las primeras muertes la noticia se seguía periodísticamente durante aproximadamente una semana, en 1983 tanto Clarín como La Nación darían lugar a la muerte de Aníbal Taranto diariamente por más de 15 días" (2010: 6). Se trata del primer hecho donde los medios darían más espacio de cobertura a la violencia que al propio partido de fútbol. A partir de allí, los incidentes ocuparán paulatinamente un lugar cada vez más destacado en las crónicas.

El aumento cuantitativo de la cobertura de la violencia en el futbol implicó la puesta en práctica de un proceso de "construcción discursiva de la amenaza", que se apoyó en ciertos modos habitualmente utilizados en la materia (COHEN,1972). Así, la presencia creciente en la prensa de los incidentes 
de violencia, incluso aquellos menores, dio inicio a la expansión de una idea de desorden, decadencia y peligro alrededor de los partidos de fútbol. La percepción social respecto a que los incidentes eran reflejo de una desintegración del orden social, operó en Argentina de forma similar a lo descripto por Hall (1978) en relación a la sociedad británica y el pequeño delito en los años 1970. Ese "pánico moral", motivado por los grupos de hinchas de fútbol organizados, se trasluce de forma brutal en el film de Enrique Carreras "Las barras bravas". Estrenado en agosto de 1985, el largometraje presenta una lectura burda y maniquea de los grupos de hinchas organizados, que sin embargo refleja potentemente las representaciones sociales del fenómeno en la época.

De la mano de la instalación de los "pánicos morales", del crecimiento real de los incidentes en los estadios a partir del inicio de la década de 1980 (ROMERO,1985) y de la consolidación de las "barras bravas" como actores ineludibles de las tribunas, aquella visión del fútbol extendida en los discursos sociales y mediáticos como una "fiesta de todos", en la cual los hechos de violencia eran algo insólito, extraño y extemporal, fue dando paso a otro tipo de concepción del espectáculo futbolístico, menos festiva y más dramática.

En el marco del escenario descripto, dos acontecimientos ocurridos en 1985 resultarán fundamentales para la definitiva instalación de la violencia en el fútbol como un problema público en Argentina. El primero es la llamada "Tragedia de Heysel", como se conoce a los sucesos ocurridos el 29 de mayo de ese año en la final de la Copa de Campeones de clubes europeos -equivalente de la actual Champions League- entre Juventus de Italia y Liverpool de Inglaterra. Debido a una avalancha ocasionada por la acción de los hooligans ingleses, 39 personas perdieron la vida en los momentos previos al inicio del partido, que se disputaba en la ciudad de Bruselas, en Bélgica. El impacto de Heysel se potenció debido a la importancia del partido $\mathrm{y}$, sobre todo, a su transmisión televisiva, de carácter masiva, pormenorizada y en directo.

El drama de Heysel se produce en un momento en que el fenómeno del hooliganismo era objeto de controversia en Inglaterra desde tiempo atrás (MURPHY, 1990) y había insistentes demandas de endurecimiento del control en los estadios formulados por la prensa. Según Mignon (1998), esos reclamos tenían dos objetivos más amplios, que excedían al mundo del fútbol: atacar la descriminalización que las leyes venían proponiendo, y velar por la "declinación moral" de la juventud inglesa. En este marco, las voces conservadoras pedían castigar a los hooligans, quienes eran vistos como los representantes de la decadencia de la juventud inglesa.

En dicho momento se inicia un proceso de cambio en las reglamentaciones sobre violencia en el espectáculo futbolístico a nivel inglés (que tendrá su culminación con el "Informe Taylor" de 1991 y las consecuencias que acarrea a nivel del Estado y del negocio del fútbol) y también europeo. A nivel continental, el Consejo de Europa (Consejo de Europa, Recomendación $\mathrm{n}^{\mathrm{a}} \mathrm{R}(84) 8$, marzo 1984) se había pronunciado meses antes de Heysel por primera vez sobre la reducción de la violencia en manifestaciones deportivas, particularmente en partidos de futbol. En ese documento se sugerían medidas de vigilancia de espectadores y de control de venta de entradas, barreras de protección en las canchas y medidas de restricción y prohibición de bebidas alcohólicas dentro de los estadios. Inmediatamente luego de Heysel, ese mismo Consejo incorporará al documento nuevas medidas de control. A partir de allí, el dispositivo de control social del hooliganismo a nivel europeo no se limitará ya a aportar respuestas al prejuicio real causado por actos delictuales, sino que se expandirá para cubrir también el riesgo potencial que representan los comportamientos "desviados" (TSOUKALA, 2009).

Heysel marca un quiebre en la reglamentación del hooliganismo porque por primera vez el fenómeno es considerado un problema de orden público que necesita la adopción de medidas específicas. Distinguiéndose de la posición moderada hasta allí adoptadas a niveles nacionales y continentales, estas disposiciones prefiguran una nueva época de control social en Europa, basadas cada vez más crecientemente en la gestión del peligro. En ese sentido, Inglaterra aplicó las primeras medidas de control de los hinchas a partir de dos métodos: la videovigilancia, y la separación de hinchas tanto dentro del estadio (a partir de la venta diferenciada de tickets, con muros humanos de policías y con barreras, grillas y otros separadores físicos) como fuera (con muros de policías custodiando a los hinchas visitantes) (SEGURA \& MURZI, 2013). 
Con el trasfondo de Heysel latente, en Argentina los cambios en el control del fenómeno de la violencia en el fútbol no llegaron sin embargo como producto de una tragedia colectiva. El "Heysel argentino" -la Puerta 12- había ocurrido 15 años antes y apenas había propiciado una ordenanza municipal luego derogada. En el país, el hecho que dio lugar a la primera política pública sobre violencia en espectáculos deportivos fue la muerte del joven hincha de Boca Adrián Scasserra, asesinado por un disparo presuntamente policial en el estadio de Independiente el 7 de abril de 1985.

La muerte de Scasserra, de 14 años, se produjo luego de una pelea de hinchas de Boca con la policía en una tribuna. El episodio conmovió al mundo del fútbol y a la política nacional, y constituyó un cimbronazo para el gobierno de Raúl Alfonsín. La violencia en el fútbol, inscripta en ese colectivo emergente de "nuevas" violencias civiles y sociales que señalábamos antes, se presentaba como un desafío para la legitimidad de un Estado democrático muy reciente y por ende aún débil y en vías de construcción. En ese contexto, la conservación y la expresión del monopolio de la violencia física podía verse como un elemento de vital importancia para el futuro político del alfonsinismo (ACOSTA, 2010), en tanto aún tallaban fuertemente los imaginarios que asociaban a los gobiernos democráticos con la debilidad y la ineficacia en los asuntos de seguridad y de "defensa" (PEGORARO, 2000).

La muerte de Scasserra puede considerarse lo que Anthony Downs (1993) denomina un "descubrimiento alarmante" en sus trabajos sobre ciclos de formación de políticas públicas. Según el autor estadounidense, existen problemas que se hallan en un estado de latencia hasta que sobreviene un hecho alarmante que los convierten en "problemas sociales". Lo más probables es que, luego de ese pico de atención, el interés por el tema vuelva a caer, y es en ese punto donde los decisores de políticas públicas deben definir si convierten al problema en una política pública o no.

A diferencia de otros casos fatídicos que, como vimos, no tuvieron un rebote en la acción estatal frente al problema, el asesinato de Scasserra propició la que se puede considerar como la primera norma estatal específica referida al fenómeno de la violencia en el fútbol. Días después del suceso, el tema fue tratado por la Comisión de Deportes del Senado y, con aprobación de todos los sectores políticos, el 30 de mayo de 1985 se sancionó la Ley $N^{\circ} 23.184$ con el objetivo de regular y punir los hechos de violencia en espectáculos deportivos. Para Alabarces, aquí se da inicio al modelo "espasmódico" que según este autor caracteriza a las políticas públicas de seguridad en el fútbol en Argentina:

Esta asociación causa-consecuencia implica la aparición de una norma de conducta: el Estado y los representantes políticos comienzan a actuar sólo como reacción espasmódica a algún caso fatal que los motiva a ocuparse del tema, sabiendo que la presencia del caso en los medios les garantiza visibilidad y flashes por unos días (2004: 29).

La sanción de esta normativa puede considerarse un punto de llegada en el que se condensan, como señala Galar (2017:70) los "relatos y narraciones que colaboran a la estabilización de los problemas públicos en tanto los dotan de legitimidad y marcan el rumbo de las intervenciones de los actores involucrados". La Ley $\mathrm{N}^{\circ} 23.184$, además de ser la primera legislación sobre regulación de la violencia en los estadios, traduce en su contenido la importancia que el fenómeno había adquirido, así como sus interpretaciones sociales dominantes.

\section{RECAPITULACIÓN Y CONCLUSIONES}

Desde los inicios del fútbol en Argentina hasta entrada la década de 1960, los hechos de violencia alrededor del fútbol fueron concebidos por los discursos mediáticos y estatales como algo ocasional, circunstancial y extemporáneo. Predominaba la idea de que eran consecuencia de lo accidental, del exceso de apasionamiento o de la acción puntual de algún hincha irracional o fuera de sus cabales.

El tránsito hacia la idea de que el fenómeno de la violencia en el fútbol es una parte constitutiva del mismo espectáculo deportivo encuentra sus primeros antecedentes frente a las muertes de Linker en 1958 y fundamentalmente de Souto en 1967. De manera embrionaria, la idea del fútbol como evento festivo, carnavalesco y familiar va a ir dando lugar a otra imagen más sombría, que se consolidaría a partir de la vuelta de la democracia en 1983.

Argentina vive su gran tragedia masiva en un estadio de fútbol en 1969, con los sucesos de la "Puerta 12", donde pierden la vida 71 espectadores. 
Sin embargo, ese hecho dramático será leído por la mirada estatal como el mero producto de fallas edilicias, infraestructurales y, en menor medida, de organización. La responsabilidad de los clubes, de las fuerzas de seguridad y de los propios hinchas no será puesta en cuestión, por lo cual la tragedia no cambiará la concepción estatal sobre las prácticas y conductas que se desplegaban en los estadios. Producto de la "Tragedia de la Puerta 12" se sanciona una reglamentación que se puede considerar el primer antecedente legislativo específico de ordenamiento de espectáculos deportivos en la Ciudad de Buenos Aires, la Ordenanza $\mathrm{N}^{\circ}$ 24.225. En su texto, la seguridad es pensada como protección física de los espectadores frente a los peligros que representan las aglomeraciones en términos de eventuales fallas estructurales, pero no frente al riesgo que supone la acción de las otras personas que concurren al estadio. Esta ordenanza municipal constituyó además el primer eslabón de una larga cadena de dispositivos jurídicos que verán la luz como respuesta inmediata a hechos trágicos -sobre todo muertes- que tienen lugar en los estadios argentinos.

Al igual que lo que sucedía en Europa - en particular en Inglaterra, donde el fenómeno del hooliganismo crecía problemáticamente -, en Argentina la violencia en el fútbol era considerada hasta los años 1980 como un problema de orden público ordinario que no ameritaba legislaciones específicas para su control, y las faltas o delitos que tenían lugar en el mundo del fútbol eran juzgados por el Código Penal general.

A partir de la vuelta del proceso democrático en 1983 esta situación se modifica, y la violencia en el fútbol comienza a ser percibida como un problema público para el Estado, debido a tres motivos principales: a) el cambio operado en los grupos de hinchas organizados hacia formas donde la violencia fue adquiriendo preponderancia, con la consolidación de la figura de las "barras bravas" crecientemente asociadas al crimen y al delito, b) la emergencia de los “pánicos morales” (COHEN, 1972) asociados a la caracterización que hacen los medios de comunicación de los hinchas de fútbol, que genera que tanto el espectáculo futbolístico como los grupos de hinchas organizados comiencen a ser percibidos como focos de amenaza y peligro, y c) los cambios a nivel social y político que motivaron el retorno de la democracia, donde a partir del progresivo declive de la violencia política que caracterizó esos años, la restitución del proceso democrático vio emerger nuevas violencias y conflictividades sociales que rápidamente se incorporaron a la agenda política como nuevos fenómenos a los que el Estado debió dar respuesta (KESSLER, 2009).

El año 1985 es central para el campo de la seguridad deportiva en Argentina, debido a que se sanciona la primera Ley Federal de regulación de la violencia en los espectáculos deportivos. Con el trasfondo de la "tragedia de Heysel" en Bélgica, que motiva un cambio sustancial en la mirada sobre el control del hooliganismo a nivel europeo, sumado al nuevo tratamiento que el Estado argentino da a las violencias civiles en el marco del reciente Estado de Derecho, a partir de la sanción de la Ley $N^{\circ} 23.184$ se produce la consolidación de la violencia en el fútbol como un problema público.

A partir de la acentuación de los "pánicos morales" asociados a los hinchas de fútbol en los discursos mediáticos y de la creciente visibilidad de las "barras bravas" como actores punibles y perjudiciales, la tradicional visión del fútbol como "fiesta popular" virará hacia una caracterización más conflictiva, violenta y problemática del espectáculo futbolístico.

La Ley $\mathrm{N}^{\circ} 23.184$, motivada por el crimen del joven Adrián Scasserra en abril de 1985, es una piedra nodal en la historia de la seguridad deportiva argentina, e inaugura muchos de los supuestos sobre los cuales se concebirá a la violencia en el fútbol en las décadas subsiguientes.

\section{BIBLIOGRAFIA}

ACOSTA, MARIA ELENA. Decisiones en materia de Seguridad durante las presidencias de Alfonsín y Menem. Presentado en V Congreso Latinoamericano de Ciencia Política. Asociación Latinoamericana de Ciencia Política, Buenos Aires, 2010.

ALABARCES, PABLO. Fútbol y Patria: el fútbol y las narrativas de la nación en la Argentina. Buenos Aires, Argentina: Prometeo, 2002.

ALABARCES, PABLO. Crónicas del Aguante. Fútbol, violencia y política. Buenos Aires: Capital Intelectual, 2004. 
ALABARCES, PABLO. Héroes, machos y patriotas: El fútbol entre la violencia y los medios. Buenos Aires, Argentina: Aguilar, 2015.

ARCHETTI, EDUARDO. Argentinian Soccer: A ritual of violence? In: The International Journal of the History of Sport, 1992, Vol.9, No. 2, p. 209-232.

ARMSTRONG, GARY; HARRIS, ROSEMARY. Soccer hooligans. In: Theory and evidence. Sociological Review, 1991, Vol. 39, No. 3, p. 427-567.

BOURDIEU, PIERRE. Programa para una sociología del deporte. En Cosas dichas, Buenos Aires, Argentina: Gedisa, 1988.

BRAUN, ROBERT; VLIEGENTHART, RENS. The Contentious fans: The impact of reppression, media coverage, grievance and aggressive play on supporters violence. International Sociology, 2008, No. 23, p. 796-818.

BROHM, JEAN-MARIE. Sociología politica del deporte, México: Fondo de Cultura Económica, 1982.

CABRERA, NICOLÁS. Gramsci, Bebote y Bullrich. La papa que calienta y no quema. Revista Voces en el Fénix, $\mathrm{n}^{\mathrm{o}}$ 58, Facultad de Ciencias Económicas, Buenos Aires: Argentina. 2016.

CAMAROTTI, ANA CLARA, JONES, DANIEL, \& DI LEO, PABLO. (Dir.) Entre dos mundos, Buenos Aires, Argentina: Teseo. 2017.

CLARKE, JOHN. The Three Rs-Repression, Rescue and Rehabilitation: Ideologies of Control for Working Class Youth ( $\left.\mathrm{N}^{\circ} .41\right)$. Centre for Contemporary Cultural Studies, University of Birmingham. 1976.

COHEN, STANLEY. Demonios populares y 'pánicos morales': delincuencia juvenil, subculturas, vandalismo, drogas y violencias, Barcelona, España: Gedisa, 1972.

DE SOUSA SANTOS, BOAVENTURA. Refundación del Estado en América Latina: Perspectivas desde una epistemología del Sur, La Paz, Bolivia: Plural Editores. 2010.

DOWNS, ANTHONY. El ciclo de atención a los problemas sociales. Problemas públicos y agenda de gobierno, México, Miguel Ángel Porrúa, 141-159. 1993.

ELIAS, NORBERT. Deporte y violencia. En Wright Mills, C. et al., Materiales de sociología crítica. Madrid, España: Genealogía del Poder, 1986.

ELIAS, NORBERT \& DUNNING, ERIC. Sport et civilisation. La violence maitrisée. Paris, Francia: Fayard. 1994.

FABBRI, ALEJANDRO \& PANNO, JUAN JOSÉ. Los juegos de fútbol. Buenos Aires, Argentina: Ci Capital Intelectual, 2008.
FRYDEMBERG, JULIO. Historia social del fútbol en Argentina. Buenos Aires: Siglo XXI, 2010.

GALAR, SANTIAGO. Problematizar el problema: apuntes para complejizar el abordaje de la inseguridad en la dimensión pública (En línea). Papeles de Trabajo (Instituto de Altos Estudios Sociales), 11(19): 61-75. 2017.

GALVANI, MARIANA et. al. A la inseguridad la hacemos entre todos, Buenos Aires: Argentina: Hekht Libros. 2010.

GARLAND, DAVID. La cultura del control: Crimen y orden social en la sociedad contemporánea. Barcelona, España: Gedisa. 2006.

GARRIGA, JOSÉ. Cartografías de la(s) violencia(s). In.: GARRIGA, JOSÉ. Violencia en el fútbol: investigaciones sociales y fracasos políticos. Buenos Aires: Godot, 2013.

GIULIANOTTI, ROBERT \& KLAUSER, FRANCISCO. Security governance and sport mega-events: Toward an interdisciplinary research agenda. Journal of Sport and Social Issues, 34(1), 49-61. 2010.

HALL, STUART. The treatment of the hooliganism in the press. En Ingham, D, Football Hooliganism, Londres: Inter action Imprint. 1978.

INGHAM, ROGER. Soccer Hooliganism. London: InterAction Imprint, 1978.

JELIN, ELIZABETH. La política de la memoria: el movimiento de derechos humanos y la construcción democrática en la Argentina. En Acuña, C. H. \& Przeworski, A. Juicio, castigos y memorias: derechos humanos y justicia en la política argentina, Buenos Aires, Argentina: Editorial Nueva Visión. 1995.

KESSLER, GABRIEL. El sentimiento de inseguridad. Sociología del temor al delito. Buenos Aires: Siglo XXI Editores. 2008.

KESSLER, GABRIEL (comp.) Seguridad y Ciudadanía: nuevos paradigmas, reforma policial y políticas innovadoras, Buenos Aires, Argentina: Edhasa. 2009.

MARSH, PETER. Aggro: The Ilusion of Violence. London: Dent., 1975.

MIGNON, PATRICK. La Passion du Football. Paris: Odile Jacob, 1998.

MURPHY, PETER; WILLIAMS, JOHN \& DUNNING, ERIC. Football on Trial: Spectator Violence and Development in the Football World, Londres, UK: Routledge.1990.

MURZI, DIEGO. Fútbol, Violencia y Estado: Un Estudio sobre las Políticas Públicas de Seguridad Deportiva en Argentina (2006 -2017). Tesis de Doctorado en Ciencias Sociales, Universidad de Buenos Aires, 2019.

MURZI, DIEGO; ULIANA, SANTIAGO \& SUSTAS, SEBASTIÁN. El Fútbol de Luto. Análisis de los Factores de Muerte y Violencia en el Fútbol Argentino. Godio, M y 
Uliana, S. (Comps), Fútbol y Sociedad. Prácticas Locales e Imaginarios Globales. Buenos Aires: EDUNTREF 2011.

MURZI DIEGO; SEGURA FERNANDO. Hacia un Mapa de las "Violencias en el Fútbol": Actores, Dinámicas, Respuestas Públicas y Desafíos en el Caso de Argentina. In: Revista de Gestión Pública, Vol.7, No. 1, p. 43-75. 2018.

PALOMINO, HECTOR; SCHER, ARIEL. Fútbol, pasión de elites y multitudes: estudio institucional de la Asociación de Fútbol Argentino (1934-1986). Buenos Aires: CISEA, 1988.

PEGORARO, JUAN. Violencia delictiva, inseguridad urbana. Nueva Sociedad, 167, 114-131. 2000.

ROMERO, AMÍLCAR. Deporte, violencia y política. Buenos Aires: Centro Editor de América Latina, 1985.

ROMERO, AMÍLCAR. Las Barras Bravas y la Contrasociedad Deportiva. Buenos Aires: Editorial Nueva América, 1994.

SEGURA, FERNANDO \& MURZI, DIEGO. Alternativas europeas comparadas de gestión de la seguridad y la violencia en los estadios de fútbol: tres enfoques y aplicaciones diferentes. ¿ Qué se puede aprender. En: Garriga, J. Violencia en el fútbol. Investigaciones sociales y fracasos políticos. Buenos Aires: Godot. 2013.

SEGURA, FERNANDO; MURZI, DIEGO. ¿Gestión de la violencia en el fútbol? Perspectivas críticas sobre Inglaterra y Bélgica. In: Revista de Gestión Pública, Vol.4, No. 1, p. 65-106. 2015.

SEGURA, FERNANDO; MURZI, DIEGO; NASSAR, BELEN. Violence and Death in the Argentinean Soccer. In: The International Review for the Sociology of Sport, OnLine-First, 1-18. 2018.

SODO, JUAN MANUEL. Dos problemas de las clasificaciones en investigaciones sobre hinchas del fútbol argentino: apuntes desde la propia experiencia, EFDeportes $\mathrm{n}^{\circ}$ 149, Buenos Aires, 2010. Recuperado de http://www. efdeportes.com/efd149/clasificaciones-sobre-hinchas-delfutbol-argentino.htm.

SODO, JUAN MANUEL. De la violencia a los ambientes de violencia: entre el doble discurso de los hinchas y el doble reduccionismo mediático. In.: GARRIGA, JOSÉ (Ed.). Violencia en el fútbol. Buenos Aires: Godot, 2013.

SOZZO, MÁXIMO. Seguridad Urbana: nuevos problemas, nuevas perspectivas. Santa Fe: Centro de Publicaciones UNL. 2009.

SUSTAS, SEBASTIÁN. Planos y lógicas de la violencia en el fútbol. Análisis y descripción de los enfrentamientos en que suceden las muertes en Argentina. En Godio, M. y Uliana, S. (comps). Fútbol y Sociedad. Prácticas locales e imaginarios globales. Buenos Aires: Eduntref. 2011.
SUSTAS, SEBASTIAN. Las violencias sentenciadas. In: GARRIGA, JOSÉ. Violencia en el Fútbol. Buenos Aires: Godot, 2013.

SZLIFMAN, JAVIER. La Fiesta Que No Fue. Un Análisis Sobre Los Medios De Comunicación Y La Violencia En El Fútbol Argentino. Buenos Aires: EFDeportes, 2010.

TOURAINE, ALAIN. ¿Qué es la democracia?, Buenos Aires, Argentina: Fondo de Cultura Económica. 1995.

TSOUKALA, ANASTASSIA. Soccer Hooliganism in Europe: Security and Civil Liberties in Balance. Houndmills, UK: Palgrave Macmillan. 2009.

ZIMMERMAN, EDUARDO. Law, Justice and State Building. Essays in the History of Judicial Institution in Nineteenth Century Latin America. London: Institute of Latin American Studies; University of London Press. 1999. 